\title{
LOS ANÁLISIS DISCURSIVOS COMO INSTRUMENTO DE TRABAJO PARA LOS ESTUDIOS CULTURALES: LA CONSTRUCCIÓN DISCURSIVA DEL NEW MAN EN UN CONSULTORIO PARA HOMBRES BRITÁNICO
}

\author{
EDUARDO DE GREGORIO GODEO \\ Universidad de Castilla - La Mancha
}

\section{RESUMEN}

En un intento de iluminar el papel del lenguaje en la configuración de las prácticas culturales, este artículo explora las posibilidades del análisis crítico del discurso como instrumento de análisis para los estudios culturales. La aplicación del modelo de análisis crítico del discurso de Fairclough a una muestra del consultorio de una revista para hombres británica sirve para examinar, a modo de ejemplo, la construcción discursiva del New Man, poniendo de relieve la función del lenguaje en la articulación de las prácticas culturales de representación y construcción de modelos de masculinidad como éste en diversas formas de cultural popular del Reino Unido.

\section{INTRODUCCIÓN}

A pesar del carácter central de nociones como lenguaje o discurso en los estudios culturales, hasta la fecha apenas se ha intentado desentrañar el papel de lo lingüístico en la articulación de las prácticas culturales. Existen, sin embargo, modelos de análisis del discurso que, partiendo de una aproximación al estudio del lenguaje en gran medida coincidente con la teorización que desde los estudios culturales se lleva a cabo sobre la configuración de las prácticas culturales, pueden resultar sumamente eficaces para poner de relieve la función del lenguaje en la constitución de la vida cultural. Pretendemos aquí dar cuenta de las posibilidades del Análisis Crítico del Discurso (en adelante ACD) como instrumento de análisis cultural. Mediante la aplicación del modelo de ACD de Fairclough $(1989,1992,1995 a, 1995 b)$ sobre una muestra del consultorio de una revista para hombres británica, examinaremos la construcción discursiva del New Man, una de las imágenes sobre la masculinidad más recurrentes en diversos vehículos de la cultura popular del Reino Unido durante las últimas décadas. Intentaremos así iluminar la función del discurso en la conformación de prácticas culturales de representación y construcción de modelos de masculinidad como éste, y el papel del lenguaje en dicho proceso. 


\section{LA AGENDA COMÚN DE LOS ESTUDIOS CULTURALES Y EL ACD}

Desde la teoría cultural contemporánea, se ha tendido a enfatizar el papel fundamental tanto del lenguaje como de las relaciones de poder en la constitución de la cultura. De un lado, ha cobrado cuerpo la idea de cultura en tanto que intercambio de significados por parte los individuos en sociedad: "Primarily, culture is concerned with the production and exchange of meanings - the 'giving and taking of meaning' - between the members of a society or group" (Hall, 1997: 2). Según admite Hall, el lenguaje va a ser un medio privilegiado para la producción e intercambio de esos significados: "Language is the privileged meaning in which we 'make sense' of things, in which meaning is produced and exchanged" (ibid: 1$)^{1}$. Por otra parte, los estudios culturales han ido configurándose como disciplina centrada en el estudio de las formas y actividades culturales en el marco de las relaciones de poder que dan cuenta de su producción, distribución y efectos sociales: "Cultural studies is concerned with the analysis of cultural forms and activities in the context of the relations of power which condition their production, circulation, deployments and, of course, effects" (Bennett, 1998: 60). Los estudios culturales han venido explorando así el significado cultural que adquieren formas de poder asociadas al género, la raza y etnicidad, el colonialismo, los nacionalismos o la globalización, por sólo citar algunos ejemplos.

Desde el campo del análisis del discurso, el ACD comparte con los Estudios Culturales la atención al lenguaje y la estructuración de las relaciones de poder en sociedad, siendo uno de sus intereses principales el desvelar el papel desempeñado por el lenguaje en los abusos sociales de poder:

Critical discourse analysis (CDA) is a type of discourse analytical research that primarily studies the way social power abuse, dominance, and inequality are enacted, reproduced and resisted by text and talk in the social and political context. Within such dissident research, critical discourse analysts take explicit position, and thus want to understand, expose and ultimately resist social inequality. (van Dijk, 2001: 352)

Partiendo de una concepción discursiva del lenguaje, el ACD postula el carácter constitutivo del lenguaje respecto a la sociedad, la cultura y las relaciones de poder: "Discourse constitutes society and culture, as well as being constituted by them. Their relationship, that is, is a dialectal one. This entails that every instance of language use makes its own small contribution to reproducing and/or transforming society and culture, including power relations" (Fairclough y Wodak, 1997: 273).

Resultan, así pues, incontestables, las coincidencias de los estudios culturales y del ACD a propósito del papel fundamental desempeñado tanto por el lenguaje como por las relaciones de poder en la conformación de la cultura. De manera próxima al compromiso político del ACD, los estudios culturales se han marcado siempre como objetivo primordial el dar visibilidad a la relación que poseen con la cultura los grupos más marginales y

\footnotetext{
${ }^{1} \mathrm{Du}$ Gay et al. aluden al proceso de representación que el lenguaje lleva a cabo en la cultura: "Culture, then, is inextricably connected with the role of meanings in society. It is what enables us to 'make sense' of things. But how does this 'meaning-making' work? Partly, we give things meanings by the way we represent them, and the principal means of representation in culture is language " (1997: 13).
}

Odisea, $n^{\circ} 4,2003$ 
menos favorecidos en el contexto de las relaciones de poder imperantes en la sociedad: "Cultural studies has been, as we might expect, most interested in how groups with least power practically develop their own readings of, and uses for, cultural products - in fun, in resistance, or to articulate their own identity" (During, 1993: 7). De otro lado, el examen del discurso, labor consustancial al ACD, no ha escapado tampoco a los objetivos de los estudios culturales. Bajo la impronta de Michel Foucault y el posestructuralismo, en la teoría cultural ha prevalecido una aproximación al discurso relativa al modo de construir y referirse a los dominios de conocimiento asociados a prácticas sociales determinadas: "Discourses are ways of referring to or constructing knowledge about a particular topic of practice: a cluster (or formation) of ideas, images and practices, which provide ways of talking about, forms of knowledge and conduct associated with, a particular topic, social activity or institutional site in society" (Hall, 1997: 6). El discurso es, según Grossberg (1997: 5), objeto primordial de los estudios culturales, si bien no tanto el discurso per se, sino los diferentes modos de articular la vida cotidiana y las formaciones de poder.

Según subrayan autores como Jaworski y Coupland, los beneficios que reporta toda consideración del lenguaje como discurso para los análisis culturales resultan innegables: "Language studied as discourse opens up countless new areas for the critical investigation of social and cultural life - the composition of cultural groups, the management of social relations, the constitution of social institutions, the perpetuation of social prejudices, and so on" (1999: 5). Sin embargo, como hemos visto, desde la teoría cultual, se ha tendido a operar con una noción bastante abstracta de discurso, difícil de aplicar a muestras concretas de lenguaje. Es aquí donde, dadas estas asunciones comunes con la teoría cultural, el ACD puede erigirse en una herramienta eficaz para desentrañar el papel desempeñado por el lenguaje en la configuración de las prácticas culturales. En efecto, hablar hoy de discurso es hablar "de una práctica social, de una forma de acción entre las personas que se articula a partir del uso lingüiśtico contextualizado ya sea oral o escrito" (Calsamiglia y Tusón, 1999: 15); y en tanto que prácticas sociales, los discursos serían a su vez prácticas culturales: "Theorists have begun to argue that because all social practices are meaningful practices, they are all fundamentally cultural" (du Gay et al., 1997: 2). De este modo, según han apuntado recientemente Barker y Galasinski con su voluntad de acercamiento entre los estudios culturales y el análisis del discurso, el análisis de discursos concretos puede y debe convertirse en objeto de los análisis culturales:

Though cultural studies has produced a large body of textual analysis and made claims regarding the social construction of gender, ethnicity, class, age, etc., it has only rarely attended to the day-to-day speech of acculturated persons. Consequently, cultural studies has been unable to show how precisely the discursive construction of cultural forms is achieved in everyday life. (2001:27)

En esta tarea, el ACD puede servir como eficiente instrumento de trabajo para descifrar la función del lenguaje en la conformación de las prácticas culturales que se desarrollan en una sociedad. 


\subsection{El modelo de Análisis Crítico de Discurso de Fairclough}

Desde su fundamentación teórica, el modelo de ACD de Norman Fairclough (1989, 1992, 1995a, 1995b) se propone desentrañar qué papel desempeña el lenguaje en la vida cultural. El lenguaje se entenderá como discurso y, en tanto que tal, como forma de práctica social: "For CLS [Critical Language Study], the conception of language we need is that of discourse, language as a form of social practice" (1989: 20). Ahora bien, Fairclough incidirá en el carácter no solamente social del discurso sino en su naturaleza cultural: "A piece of discourse is embedded within sociocultural practice at a number of levels; in the immediate situation, in the wider institution or organization, and at a societal level" (1995a: 97).

Frente a otras tradiciones de $\mathrm{ACD}^{2}$, el modelo de Fairclough - del que aquí nos servimos- parte de una aproximación simultáneamente tripartita al discurso, esto es, como texto, como interacción entre individuos o práctica discursiva que da lugar a procesos de producción, interpretación, distribución y consumo de los discursos en sociedad, y como práctica o acción sociocultural: "Discourse, and any specific instance of dicursive practice, is seen as simultaneously (i) a language text, spoken or written, (ii) discourse practice (text production and text interpretation), (iii) sociocultural practice" (Fairclough, 1995a: 97). El análisis de los discursos concretos tendrá en cuenta la descripción de la dimensión textual del discurso; la interpretación de la interacción o práctica discursiva, atendiendo a los procesos de producción, interpretación, y consumo de esos discursos; y la explicación del discurso en tanto que práctica o acción sociocultural que emana de una matriz social y tiene una ideología subyacente.

\section{CASO PRÁCTICO: UN EJEMPLO DE CONSTRUCCIÓN DEL NEW MAN EN EL DISCURSO DE UNA SECCIÓN DE CONSULTA MASCULINA}

Como ya se ha señalado, a raíz de la fuerte huella de Foucault (v.g. 1972) en la teoría cultural, se ha consolidado una idea de los discursos en tanto que modos de construir los objetos de conocimiento y las prácticas sociales: "For Foucault, discourses are particular ways of organising knowledge in the context of serving specific types of power relationships" (Strinati, 1995: 249). Regulados por las relaciones de poder en que emergen los discursos, éstos van a generar modelos de identidad o posiciones de sujeto con las que los individuos concretos negociarán su identidad personal, y desde las cuales darán sentido a la realidad ${ }^{3}$. Junto a otras dimensiones de la identidad del individuo -como la etnicidad, la clase social o la edad-, los discursos van a dar lugar a posiciones de sujeto desde la que los individuos activen su identidad de género: "Gender can be thought of as a particular discourse (...) Gender is inscribed in the subject along with other discourses, such as those of ethnicity, class and sexuality, in a variety of cultural practices" (van Zoonen, 1992: 20).

\footnotetext{
${ }^{2}$ Fairclough y Wodak (1997) y van Dijk (2001) ofrecen exhaustivos repasos de otras tradiciones de ACD. ${ }^{3}$ Las posiciones de sujeto surgen de la facultad de los discursos de construir los sujetos a los que envuelven dotando a los individuos de modelos de identidad que éstos vienen a activar: "Social subjects can be conceived of in terms of the positioning of people progressively over a period of years (...) The social subject is thus constituted as a particular configuration of subject positions" (Fairclough, 1989: 103).
}

Odisea, $n^{\circ} \quad 4,2003$ 
En concreto, Sarah Mills admite que es posible la construcción de discursos concretos sobre la masculinidad y la feminidad: "We can assume that there is a set of discourses of femininity and masculinity, because women and men behave within a certain range of parameters when defining themselves as gendered subjects" (1997: 17).

En este contexto, la representación de lo masculino en diversas formas de la cultura popular británica contemporánea ha ido generando la imagen de un "hombre nuevo" o New Man como modelo de masculinidad que incorpora rasgos como una renovada atención a su dimensión emocional, la mayor complacencia de los deseos y requerimientos femeninos, o -como desvelará nuestro análisis- el mayor cuidado de su estética personal: "The New man arose as a primarily media-driven phenomenon in the 1980s (...) a new form of masculinity that was more caring, nurturing and sensitive - or, alternatively, more narcissitic, passive and introspective" (Edwards, 1997: 39). Se trataría de una posición de sujeto dentro de los discursos sobre lo masculino generados desde los medios de comunicación de masas del Reino Unido desde la década de los ochenta.

El análisis crítico del discurso de una muestra -transcrita en el apéndice final- extraída de la sección de consulta de la revista británica de interés general para hombres Later nos servirá así para poner de relieve cómo se representa discursivamente la imagen del New $\mathrm{Man}^{4}$, dejando patente la función primordial del lenguaje en la construcción de este modelo de masculinidad. Con ello, estaremos partiendo de la función que Fairclough otorga al discurso en la representación de las identidades y en la construcción de las mismas: "Discourses do not just reflect or represent social entities and relations, they construct or 'constitue' them; different discourses constitue key entities in different ways, and position people in different ways as social subjects" (1992: 3-4).

De entrada, la estructuración del discurso a nivel textual evidencia una organización del léxico en torno a esquemas de clasificación relativos a la preocupación del lector que escribe al consultorio por su imagen personal (spend more time in front of the miror; worrying about how I look; look good [tres veces]), y el narcisismo en que hombres como él acaban cayendo como resultado (vain; narcissistic men; the story of Narcissus; falling in love with his reflection; love themselves). No faltan tampoco vocablos relativos a los efectos negativos de la excesiva preocupación masculina por el cuidado de la estética personal (probem; a real turn off; make too much of a fuss; condemned to isolation).

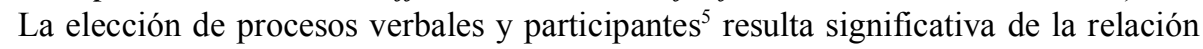
que existe entre el cuidado entre los varones de su estética personal y su atención a los

\footnotetext{
4 Por breve que sea, cualquier texto puede entenderse como una forma de discurso con sus tres dimensiones constitutivas: como pura textualidad, como interacción discursiva, y como práctica sociocultural: "Any part of any text can fruitfully be examined in terms of the co-presence and interaction of these constitutive processes" (Fairclough, 1995a: 6). El propio Fairclough (1989: 172-196) 1leva a cabo la práctica de analizar la construcción discursiva de posiciones de sujeto concretas a través del análisis de muestras textuales individuales; es el caso del examen del discurso thatcherista en una entrevista radiofónica.

${ }^{5}$ Nuestra experiencia de la realidad se constituye, según Halliday (1995: 106), bajo la forma de procesos o acciones en curso. Estos procesos, que llevan aparejados unos participantes en los mismos, pueden ser materiales, que indican acción (v.g. do, act); relacionales, relativos al mundo de las relaciones abstractas de clasificación e identificación (v.g. be, become); mentales, referentes a la cognición (v.g. see, feel, think); de tipo verbal (v.g. say); y de comportamiento, característicos del comportamiento fisiológico o psicológico humano (v.g. dream, laugh, sing).
} 
requerimientos femeninos. El narcisismo del lector lleva a representarlo como participante identificado ${ }^{6}$ de procesos relacionales con los que se describe a sí mismo (...how I look...; ...I'm so vain; ...the fact that I look good; ...you looking good; are you making yourself look good...?). Naturalmente, la construcción del lector como narcisista se lleva a cabo también mediante procesos materiales y de comportamiento que describen su modo de obrar (...that I spend more time in front of the mirror and worrying about...), y procesos mentales, que dan cuenta de la dimensión emocional de la actitud del lector ( $I$ know she also...; ...not if you make too much of a fuss about it). Por otra parte, su novia se construye como el participante que, generando la señal comunicativa correspondiente en los procesos de tipo verbal ${ }^{7}$, explica a su compañero la opinión que le merece su actitud ante el cuidado de su estética (My girlfriend told me...; She says...). Cuando expresa estas preferencias, también se la construye como sujeto ${ }^{8}$ de procesos mentales (...she also likes the fact that I look good; maybe your girlfriend likes you looking good...).

Los enunciados negativos se han seleccionado para presuponer un tipo de hombre que se preocupa más por sí mismo y su apariencia personal que por su propia pareja (Maybe your girlfriend likes you looking good, but not if you make too much of a fuss about it; Many women give up on narcissistic men, as they feel they can never love them as much as they love themselves) $)^{9}$.

La utilización de ciertos rasgos de modalidad y actos de habla viene a matizar el modo en que el asesor hace consciente al lector de los riesgos de preocuparse en exceso por su imagen. Maybe viene así a cuestionar de alguna manera la premisa de que parte el lector de que se cuida tanto porque esto le gusta a su novia (First, maybe your girlfriend likes you looking good, but not...). Por medio de un acto de habla indirecto ${ }^{10}$ (Are you making yourself look good for her or for yourself?), el asesor le hace así consciente de que quizás se cuide más para sí mismo que para su novia. Sin embargo, no duda en mostrarse tajante al advertirle de estos riesgos por medio del acto de habla directivo ${ }^{11}$ correspondiente (So be warned), significativamente introducido por so como marcador discursivo consecutivo (Schiffrin, 1987: 191-227) con el que concluye su argumentación al respecto.

En el campo de la cohesión textual, el recurrir a conectores comparativos sirve para construir un tipo de hombre para el que la atención a la estética personal da lugar a una verdadera relación de competitividad con sus parejas, (My girlfriend told me that I spend

\footnotetext{
${ }^{6}$ De acuerdo con Halliday (1994: 120), frente al participante identificado (carrier), habría un atributo (attribute), v.g. Your story [carrier] sounds [proceso relacional] complete nonsense [atributo].

${ }^{7}$ Halliday denomina a este participante sayer: "The Sayer can be anything that puts out a signal (...) [v.g.] the light in the light says stop" (1994: 140).

${ }^{8}$ Senser, según la terminología de Halliday (1994: 117).

9 "A presupposition is something the speaker assumes to be the case before making an utterance" (Yule, 1996: 25). Según afirma Fairclough, los enunciados negativos presuponen universos idológicos que, aun negados, quedan patentes en el discurso: "negative sentences carry special types of presupposition which also work intertextually, incorporating other texts only in order to contest them or reject them" (1992: 121-122).

${ }^{10}$ Un acto de habla es "la emisión de un ENUNCIADO en un CONTEXTO dado para llevar a cabo los fines de la INTERACCIÓN COMUNICATIVA" (Alcaraz y Martínez, 1997: 14). En los actos de habla indirectos, existe una relación indirecta entre la estructura y la función comunicativa de un enunciado, por ejemplo, utilizar una estructura interrogativa -como la del texto analizado- para realizar una aseveración. ${ }^{11}$ El hablante usa los actos directivos para intentar que el receptor haga algo, v.g. pedir, rogar, advertir.
} 
more time in front of the mirror and worrying about how I look than she does; many women give up on narcissistic men as they feel they can never love them as much as they love themselves). En este sentido, tanto en la pregunta del lector (She says it's a real turn off that I'm so vain. But I also know she also likes the fact that I look good) como en la respuesta del asesor (Maybe your girlfriend likes you looking good but not if you make too much of a fuss about it), la elección del conector de oposición o adversativo but manifiesta las contrariedades ligadas al excesivo cuidado masculino por la estética personal. Asimismo, la abundancia de formas de referencia personal resulta elocuente del alcance que dimensiones como la preocupación estética de los varones tienen en la configuración de las relaciones personales entre hombres y mujeres (v.g. My girlfriend told me that $\underline{I}$ spend more time in front of the mirror and worrying about how I look than she does; many women give up on narcissistic men as they feel they can never love them as much as they love themselves). La reiteración de ciertos vocablos como problem (tres veces), girlfriend (dos) y look good (tres) incide en lo problemático que el excesivo narcisismo masculino puede resultar en la vida en pareja.

La selección de los rasgos textuales descritos se puede interpretar en función del proceso de construcción de una práctica discursiva centrada sobre las tribulaciones que en la vida de pareja de muchos hombres ocasiona el excesivo mimo con que cuidan su estética personal. Este tipo de discurso se produce según las convenciones del género de las secciones de consulta de la prensa escrita. Una forma del discurso del asesoramiento como es esta interacción entre un lector y un asesor adquiere la divulgación característica del discurso periodístico merced a la publicación de la consulta individual en una revista. Los editores del consultorio de la revista masculina Later han publicado la consulta analizada en tanto que representativa de los conflictos, los intereses y el sistema de valores de los lectores masculinos ideales de esta publicación. De hecho, siguiendo una práctica editorial común que describe McCracken (1993: 57) a propósito de los consultorios de la prensa, la consulta original puede haber sido manipulada, e incluso creada artificialmente, de acuerdo con la ideología de la revista. Desde Later se presupone, por tanto, la existencia de muchos otros hombres sumamente preocupados por el cuidado de su estética personal que viven conflictos similares al de este "hombre nuevo" que emerge desde este discurso. Los lectores individuales tendrán que negociar así su identidad individual con el sistema de valores asociado al significado de lo masculino que se está construyendo desde la revista $^{12}$. El elevado consumo en el Reino Unido de revistas como Later -y otras como FHM, Maxim, Men's Health, Arena o Esquire- da cuenta de la elevada distribución de las imágenes de lo masculino que desde secciones como ésta se proyectan entre los lectores ${ }^{13}$.

Para explicar la matriz social de prácticas culturales de representación de esta nueva imagen de la masculinidad o New Man en formas de cultura popular como esta revista, hay

\footnotetext{
${ }^{12} \mathrm{Si}$ el discurso en general creará posiciones de sujeto a partir de las cuales los individuos van a construir su identidad, el discurso periodístico en particular producirá, según Reah (1998: 40), lectores ideales sobre los que se presupondrán determinados sistemas de valores. De acuerdo con McLoughlin (2000: 67), la construcción de imágenes ideales de lo masculino o lo femenino a la que los lectores han de aspirar se pone especialmente de relieve en las revistas de interés general masculinas y femeninas.

13 Jackson, Stevenson y Brooks (2001: 30) dan cifras, para el año 2000, de entre 100.000 y 700.000 ejemplares semestrales.
} 
que remitirse a la crisis de los modelos de masculinidad tradicionales producida por los cambios socioeconómicos y la crítica feminista en el Reino Unido: "The emergence of the 'new man' as a cultural frame of reference has been linked to changes in other social and economic practices [...] a further (overdetermining) factor is the feminist movement and its critique of men and male psychology" (Edley y Wetherell, 1997: 204). A ello habría que añadir, según Rutherford, el fuerte impacto de la cultura consumista de las últimas décadas sobre los varones: "It [masculinity] has become aspirational and more narcissistic, affected by the consumer market and the purchasing of sytle and appearance" (1988: 39). Se explica así la ideología subyacente en el discurso analizado, caracterizada por el narcisismo y la preocupación por la estética personal extrema de muchos varones, ligado al deseo de satisfacer los deseos femeninos, por ejemplo, en el seno de la vida en pareja. Ello es una de las notas definitorias del aparato ideológico que envuelve la construcción de discursos sobre el New Man como el activado en el fragmento del consultorio examinado: "What was distinctive about the "new man" imagery was [...] the sanctioning of a highly staged narcissism through the codes of dress and grooming" (Nixon, 1996: 202).

\section{REFLEXIONES FINALES}

Huelga decir que sería preciso analizar una selección de muestras más amplia de consultorios de revistas como Later para colegir que la construcción del New Man en este género va a manifestar siempre los rasgos lingüísticos aquí reseñados. Nuestro objetivo ha sido más bien dar cuenta de la eficacia del ACD como instrumento de trabajo de los análisis culturales mediante el examen de un caso práctico. El análisis de la muestra individual ha permitido observar de manera diáfana la construcción del discurso sobre el New Man, cosa que no hubiera sido posible de haberse analizado un elevado número de muestras, que no habríamos podido reproducir a modo de apéndice. Las prácticas de representación y de construcción de imágenes o modelos de masculinidad -posiciones de sujeto- como ésta en formas de cultura popular británica como la revista Later tienen una dimensión discursiva. El ACD ha ayudado a descifrar cómo actúa el lenguaje en esas prácticas culturales y las relaciones de poder que las determinan. Los rasgos lingüísticos destacados (léxico, negaciones, procesos verbales, actos de habla, cohesión, etc.) se encuentran al servicio del sistema de valores que caracteriza las prácticas de representación del New Man en el Reino Unido, y contribuyen a la construcción de su universo ideológico en artefactos culturales como las secciones de consulta de las revistas para hombres. La aplicación del modelo de Fairclough ha puesto así de manifiesto como se articula este discurso desde el estrato de lo eminentemente textual hasta el nivel de la acción sociocultural en la que el texto concreto viene a inscribirse.

APÉNDICE: MUESTRA ANALIZADA (LATER, SEPTIEMBRE 1999, P. 26)

Q: My girlfriend told me that I spend more time in front of the mirror and worrying about how I look than she does. She says it's a real turn off that I'm so vain. But I know she also likes the fact that I look good, so what's the problem?

THE EXPERT

Odisea, $n^{\circ} \quad 4,2003$ 
Your problem can be broken down into two parts. First, maybe your girlfriend likes you looking good, but not if you make too much of a fuss about it. This leads onto the second part of the problem. Are you making yourself look good for her or for yourself? The sad part of the story of Narcissus was that by falling in love with his reflection he was condemned to isolation. Many women give up on narcissistic men as they feel that they can never love them as much as they love themselves. So be warned.

\section{BIBLIOGRAFÍA}

Alcaraz, E. y M. A. MarTínez. 1997. Diccionario de Lingüística Moderna. Barcelona: Ariel.

Barker, C. y D. Galasinski. 2001. Cultural Studies and Discourse Analysis. A Dialogue on Language and Identity. Londres: Sage.

Bennett, T. 1998. Culture: A Reformer's Science. Londres: Sage.

Calsamiglia, H. y A. Tusón. 1999. Las Cosas del Decir. Manual de Análisis del Discurso. Barcelona: Ariel.

Du Gay, P., et al. 1997. Doing Cultural Studies. The Story of the Sony Walkman. Londres: Sage/The Open University.

DurING, S. 1993. “Introduction”. The Cultural Studies Reader. Ed. S. During. Londres/Nueva York: Routledge. 1-25.

EdLey, N. y M. WeTHERELL. 1997. "Jockeying for position: the construction of masculine identities". Discourse \& Society 8, 2: 203-217.

FAirclough, N. 1989. Language and Power. Londres: Longman.

. 1992. Discourse and Social Change. Cambridge: Polity.

. 1995a. Critical Discourse Analysis: The Critical Study of Language. Londres: Longman. . 1995b. Media Discourse. Londres: Edward Arnold.

Fairclough, N. y R. Wodak. 1997. “Critical discourse analysis”. Discourse as Social Interaction. Ed. T. van Dijk. Londres: Sage. 258-284.

Foucault, M. 1972. The Archaeology of Knowledge. Londres: Tavistock.

Grossberg, L. 1997. Bringing It All Back Home: Essays on Cultural Studies. Durham: Duke University Press.

Hall, S. 1997. "Introduction". Representation. Cultural Representations and Signifying Practices. Ed. S. Hall. Londres: Sage/The Open University. 1-11.

Halliday, M.A.K. 1994. An Introduction to Functional Grammar. Londres: Edward Arnold.

Jackson, P., N. Stevenson y K. Brooks. 2001. Making Sense of Men's Magazines. Cambridge: Polity. 
JaWORSKi, A. y N. COUPLAND 1999. "Perspectives on discourse analysis". The Discourse Reader. Eds. A. Jaworski y N. CouPLAND. Londres/Nueva York: Routledge. 1-44.

McCracken, E. 1993. Decoding Womens’ Magazines. Nueva York: St. Martin's Press.

McLoughlin. 2000. The Language of Magazines. Londres/Nueva York: Routledge.

Mills, S. 1997. Discourse. Londres/Nueva York: Routledge.

Nixon, S. 1996. Hard Looks. Masculinity, Spectatorship \& Contemporary Consumption. Londres: University College Press.

REAH, D. 1998. The Language of Newspapers. Londres/Nueva York: Routledge.

Rutherford, J. 1988. “Who’s that man?”. Male Order. Unwrapping Masculinity. Eds. R. CHAPMAn y J. Rutherford. Londres: Lawrence \& Wishart. 21-67.

Schiffrin, D. 1987. Discourse Markers. Cambridge: Cambridge University Press.

Strinati, D. 1995. An Introduction to Theories of Popular Culture. Londres/Nueva York: Routledge.

VAN DiJK, T. 2001. "Critical discourse analysis". The Handbook of Discourse Analysis. Eds. D. Schiffrin, D. Tannen y H. E. Hamilton. Oxford: Blackwell. 352-371.

VAN ZoOnen, L. 1992. "Feminist theory and information technology". Media, Culture and Society 14: 9-29.

Yule, G. 1996. Pragmatics. Cambridge: Cambridge University Press. 